\title{
Correction to: Experimental analysis of dynamic and static mechanical properties of deep-thick anhydrite cap rocks under high stress conditions
}

\author{
Shuai Yin ${ }^{1,2}$. Runcheng Xie ${ }^{3,4}$
}

Published online: 19 February 2019

(c) Springer-Verlag GmbH Germany, part of Springer Nature 2019

\section{Correction to: Carbonates and Evaporites \\ https://doi.org/10.1007/s13146-018-0450-1}

The original publication of the article does not indicate the second affiliation of the author Shuai Yin

The second affiliation should be:

Shandong Provincial Key Laboratory of Depositional Mineralization \& Sedimentary Minerals (Shandong University of Science and Technology), Qingdao, Shandong 266590, China)

Publisher's Note Springer Nature remains neutral with regard to jurisdictional claims in published maps and institutional affiliations

The original article can be found online at https://doi.org/10.1007/ s13146-018-0450-1.

Shuai Yin

speedysys@163.com

1 School of Earth Science and Engineering, Xi' an Shiyou University, Xi' an 710065, China

2 Shandong Provincial Key Laboratory of Depositional Mineralization \& Sedimentary Minerals, Shandong University of Science and Technology, Qingdao 266590, Shandong, China

3 State Key Lab of Oil and Gas Reservoir Geology and Exploitation, Chengdu University of Technology, Chengdu 610059, China

4 College of Energy Resource, Chengdu University of Technology, Chengdu 610059, Sichuan, China 